\title{
Multipartite entanglement detection for hypergraph states
}

\author{
M. Ghio ${ }^{1}$, D. Malpetti ${ }^{2}$, M. Rossi ${ }^{3}$, D. Bruß ${ }^{4}$, and C. Macchiavello ${ }^{5}$ \\ ${ }^{1}$ Scuola Normale Superiore, Piazza dei Cavalieri 7, 56126 Pisa, Italy \\ ${ }^{2}$ Laboratoire de Physique, CNRS UMR 5672, Ecole Normale Supérieure de Lyon, \\ Universite de Lyon, 46 Allée d'Italie, Lyon, F-69364, France \\ ${ }^{3}$ Dipartimento di Fisica and INFN-Sezione di Pavia, Via Bassi 6, 27100 Pavia, Italy \\ ${ }^{4}$ Institut für Theoretische Physik III, Heinrich-Heine-Universität Düsseldorf, D-40225 Düsseldorf, Germany and \\ ${ }^{5}$ Dipartimento di Fisica and INFN-Sezione di Pavia, Via Bassi 6, 27100 Pavia, Italy
}

\begin{abstract}
We study the entanglement properties of quantum hypergraph states of $n$ qubits, focusing on multipartite entanglement. We compute multipartite entanglement for hypergraph states with a single hyperedge of maximum cardinality, for hypergraph states endowed with all possible hyperedges of cardinality equal to $n-1$ and for those hypergraph states with all possible hyperedges of cardinality greater than or equal to $n-1$. We then find a lower bound to the multipartite entanglement of a generic quantum hypergraph state. We finally apply the multipartite entanglement results to the construction of entanglement witness operators, able to detect genuine multipartite entanglement in the neighbourhood of a given hypergraph state. We first build entanglement witnesses of the projective type, then propose a class of witnesses based on the stabilizer formalism, hence called stabilizer witnesses, able to reduce the experimental effort from an exponential to a linear growth in the number of local measurement settings with the number of qubits.
\end{abstract}

\section{INTRODUCTION}

Quantum hypergraph states were recently introduced [4, 5] in order to study a family of multi-qubit entangled states that generalise the notion of graph states [6], central in various aspects of quantum information, such as measurement-based quantum computation and quantum error correction. This family of states can also be described as locally maximally entangleable (LME) states for the particular value $\pi$ of the phase [7]. Quantum hypergraph states were shown to play a central role in many well known quantum algorithms [3, 8] and to provide extreme violation of local realism 9], leading to applications in quantum metrology and measurement-based quantum computation.

On the other hand, multipartite entanglement is a precious resource in various quantum information processing tasks, such as for example secret sharing [10], multipartite quantum key distribution [11], distributed dense coding [12], and some quantum algorithms [1, 3]. Studying multipartite entanglement properties of quantum states is therefore of fundamental interest.

In this work we study the multipartite entanglement properties of hypergraph states and the possibility of detecting multipartite entanglement via witness operators. The paper is organised as follows. In Sect. [II we recall some notions about hypergraph states and multipartite entanglement, that will then be used in the rest of the paper. In Sect. III we present an analytical procedure to derive the multipartite entanglement content for hypergraph states with a single hyperedge of maximum cardinality, for those with all possible hyperedges of cardinality equal to $n-1$ and for those with all possible hyperedges of cardinality greater than or equal to $n-1$. We also derive a lower bound to the multipartite entanglement of a generic hypergraph state. In Sect. IV we construct entanglement witness operators of two types and analyse their efficiency in terms of number of local measurement settings required. We end the paper with a summary of the results and some concluding remarks in Sect $\mathrm{V}$.

\section{PRELIMINARIES}

In this section we define quantum graph and hypergraph states. We then recall some fundamentals of the theory of quantum entanglement, introducing multipartite entanglement, the entanglement measure we will make use of in the rest of our work, and focusing on entanglement detection via entanglement witness operators.

\section{A. Quantum hypergraph states}

We define quantum hypergraph states following the approach of [5]. For a complete review on graph states we refer to [13].

Definition II.1 (Hypergraph state - Operational definition). Let $H=(V, E)$ be a hypergraph of order $n$. To each vertex $i$ we associate a qubit $q_{i}$ for $i=1,2, \ldots n$, thus associating an $n$-qubit quantum system $Q=\left\{q_{i}\right\}_{i=1}^{n}$ to the $n$-order hypergraph $H$.

We then define the hypergraph state $|H\rangle$ associated to hypergraph $H$ as the following $n$-qubit pure quantum state

$$
|H\rangle:=\prod_{k=1}^{n} \prod_{e \in E,|e|=k} C_{k}^{e}|+\rangle^{\otimes n}
$$

where $C_{k}^{e}$ is the $k$-qubit controlled-Z gate acting on the $k$ qubits connected by the $k$-hyperedge $e$ and $|+\rangle=$ $\frac{|0\rangle+|1\rangle}{\sqrt{2}}$ is a superposition of the computational basis states. The action of the control gate $C_{k}^{e}$ is defined as 


$$
C_{k}^{\left(i_{1}, i_{2}, \ldots i_{k}\right)}=\mathbb{I}^{(j)} \otimes(\mathbb{I}-\mathbb{P})+\sigma_{z}^{(j)} \otimes \mathbb{P}
$$

for all $j=i_{1}, i_{2}, \ldots i_{k}$, where $\mathbb{P}$ is the projector onto the state $|11 \ldots 1\rangle^{\left(i_{1}, i_{2}, \ldots \hat{j}, . . i_{k}\right)}$ and the notation $\hat{i}$ means that index $i$ is not included. Here, $\sigma_{z}^{(j)}$ is the Pauli- $z$-operator of vertex $j$. Hypergraph states with all hyperedges of the same cardinality $k$ are called $k$-uniform. Graph states are a particular case of $k$-uniform hypergraph states with $k=2$.

Hypergraph states, just like graph states, allow for an equivalent definition based on a generalized stabilizer formalism. However, differently from the graphs' stabilizers [13], we point out that the generalized stabilizers are no more local observables.

Definition II.2 (Hypergraph state - Stabilizer definition). We define the hypergraph state $|H\rangle$ associated to the hypergraph $H$ with $n$ vertices as the unique eigenvector with eigenvalue 1 of the set of $n$ operators $\left\{K_{i}\right\}_{i=1}^{n}$ defined as

$$
K_{i}:=\sigma_{x}^{(i)} \otimes \prod_{k=1}^{n} \prod_{e \in N(i),|e|=k-1} C_{k-1}^{e}
$$

where $\sigma_{x}^{(i)}$ is the Pauli-x-operator of vertex $i$ and $N(i)$ denotes the neighbourhood of vertex $i$.

The operators $\left\{K_{i}\right\}_{i=1}^{n}$ are called generalized stabilizer operators of hypergraph state $|H\rangle$; they are hermitian operators generating an Abelian group $\Sigma_{n}$ of $2^{n}$ elements [5]. The stabilizers and their compositions are hermitian operators.

Given any $n$-qubit hypergraph state $|H\rangle$, we introduce the hypergraph state basis, generalizing the graph state basis [13], with respect to which the stabilizer operators are simultaneously diagonalizable.

Proposition II.1 (Hypergraph state basis). Let $|H\rangle$ be an n-qubit hypergraph state and $\left\{K_{i}\right\}_{i=1}^{n}$ the set of its stabilizer operators. Then the following set of $2^{n}$ states

$$
\mathcal{B}_{n}:=\left\{\left|\phi_{s}\right\rangle:=\sigma_{z}^{s}|H\rangle \equiv \sigma_{z}^{s_{1}} \otimes \sigma_{z}^{s_{2}} \otimes \ldots \sigma_{z}^{s_{n}}|H\rangle\right\}_{s=0}^{2^{n}-1}(4)
$$

where $s$ is a binary number composed of bits $s_{1}, s_{2}, \ldots s_{n}$, forms a basis for the $n$-qubit Hilbert space $\mathcal{H}_{n} \simeq \mathbb{C}^{n}$. Moreover, stabilizer operators $\left\{K_{i}\right\}_{i=1}^{n}$ are simultaneously diagonalizable with respect to this basis

$$
K_{i}\left|\phi_{s}\right\rangle=(-1)^{s_{i}}\left|\phi_{s}\right\rangle
$$

and $\left\langle\phi_{s} \mid \phi_{t}\right\rangle=\delta_{s, t}$.
Proof. We begin by proving the statement in the particular case of an $n$-qubit hypergraph state $|H\rangle$ with only one $n$-hyperedge; this allows for a very simple representation of its stabilizer operators $K_{i}$ for $i=1,2, \ldots n$ :

$$
K_{i}=\sigma_{x}^{(i)} \otimes C_{n-1}^{(1,2, \ldots \hat{i}, \ldots n)} .
$$

We first prove that stabilizer operators $K_{i}$ of this form commute with the Pauli matrices $\sigma_{z}^{(j)}$ whenever $j \neq i$, whereas they anticommute when $i=j$. In order to evaluate the action of the stabilizer operator $K_{i}$ on qubit $j$ when $i \neq j$, we make use of expression (2) and get

$$
K_{i}=\sigma_{x}^{(i)} \otimes \mathbb{I}^{(j)} \otimes(\mathbb{I}-\mathbb{P})+\sigma_{x}^{(i)} \otimes \sigma_{z}^{(j)} \otimes \mathbb{P} .
$$

Since $\sigma_{z}^{(j)}$ commutes both with $\mathbb{I}^{(j)}$ and with itself, the commutativity is immediately verified. Instead when the two indexes coincide, a negative sign, due to the anticommutativity of the Pauli matrices, appears, namely

$$
\begin{aligned}
K_{i} \sigma_{z}^{(i)} & =\left(\sigma_{x}^{(i)} \otimes C_{n-1}^{(1,2, \ldots \hat{i}, \ldots n)}\right) \sigma_{z}^{(i)} \\
& =-\sigma_{z}^{(i)}\left(\sigma_{x}^{(i)} \otimes C_{n-1}^{(1,2, \ldots i, \ldots n)}\right) \\
& =-\sigma_{z}^{(i)} K_{i} .
\end{aligned}
$$

It follows that

$$
\begin{aligned}
K_{i}\left|\phi_{s}\right\rangle & =K_{i} \sigma_{z}^{s}|H\rangle \\
& =(-1)^{s_{i}} \sigma_{z}^{s}|H\rangle \\
& =(-1)^{s_{i}}\left|\phi_{s}\right\rangle .
\end{aligned}
$$

This same reasoning applies to a generic stabilizer: it suffices to recall that any stabilizer operator $K_{i}$ may be written as the composition of $\sigma_{x}^{(i)} \otimes \mathbb{I}_{n-1}^{(1,2, \ldots \hat{i}, \ldots n)}$ with $k$-controlled gates of the form $\mathbb{I}_{1}^{(i)} \otimes C_{k}^{\left(i_{1}, i_{2}, \ldots i_{k}\right)} \otimes \mathbb{I}_{n-k-1}$.

We finally check the orthonormality relation. Let $0 \leq$ $s, t \leq 2^{n}-1$ be two different binary numbers $s \neq t$, then there exists at least one index $i$ such that $s_{i} \neq t_{i}$, say $s_{i}=$ 1 and $t_{i}=0$. Then $K_{i}\left|\phi_{s}\right\rangle=(-1)^{s_{i}}\left|\phi_{s}\right\rangle=-\left|\phi_{s}\right\rangle$, while $K_{i}\left|\phi_{t}\right\rangle=(-1)^{t_{i}}\left|\phi_{t}\right\rangle=\left|\phi_{t}\right\rangle$, which means that $\left|\phi_{s}\right\rangle$ and $\left|\phi_{t}\right\rangle$ belong to two different, thus orthogonal, eigenspaces.

Just like for the projector on a graph state [13], as a consequence of Proposition II.1. it can be proved that, given a hypergraph state $|H\rangle$, the projector $|H\rangle\langle H|$ may be represented both in terms of the stabilizers $\left\{K_{i}\right\}_{i=1}^{n}$ and of the elements of the stabilizer group $\Sigma_{n}$ [14] as

$$
|H\rangle\langle H|=\frac{1}{2^{n}} \sum_{\sigma \in \Sigma_{n}} \sigma=\prod_{i=1}^{n} \frac{\mathbb{I}+K_{i}}{2} .
$$




\section{B. Entanglement measures and entanglement detection}

In this work we are interested in completely or fully entangled quantum states of multipartite quantum systems, hence in genuine multipartite entanglement. We remind the reader that the mixed state of a multipartite quantum system is said to be completely or fully entangled if it cannot be written as a convex combination of projectors onto states that are biseparable with respect to any bipartition, even allowing for different bipartitions in the same decomposition. For a complete review of the theory of entanglement and the problem of entanglement detection we mainly refer to [15] and [16]. Here, we first study the entanglement properties of quantum hypergraph states, and then apply the entanglement results to the construction of entanglement witness operators for the detection of genuine multipartite entanglement in the neighbourhood of a given hypergraph state.

Definition II.3 (Bipartite entanglement - Multipartite entanglement). Let $\left|\psi_{n}\right\rangle \in \mathcal{H}_{n}$ be the pure state of a composite quantum system composed of $n$ subsystems $\{1,2, \ldots n\}$. Let $A B$ be a possible bipartition of the $n$ subsystems with $A=\{1,2, \ldots k\}$ and $B=\{k+1, \ldots n\}$ for some $1 \leq k<n$. We define the bipartite entanglement of the state with respect to bipartition $A B$ as

$$
\begin{aligned}
E^{A B}\left(\left|\psi_{n}\right\rangle\right) & :=1-\max _{\left|\phi^{A}\right\rangle\left|\phi^{B}\right\rangle} \mid\left\langle\phi^{A}\left|\left\langle\phi^{B} \mid \psi_{n}\right\rangle\right|^{2}\right. \\
& \equiv 1-\alpha^{A B}\left(\left|\psi_{n}\right\rangle\right)
\end{aligned}
$$

where the maximum is taken over all pure biseparable states $\left|\phi_{k}^{A}\right\rangle\left|\phi_{n-k}^{B}\right\rangle$.

We define the state's multipartite entanglement as its minimum bipartite entanglement $E^{A B}\left(\left|\phi_{n}\right\rangle\right)$ with respect to all possible bipartitions $A B$ :

$$
\begin{aligned}
E\left(\left|\psi_{n}\right\rangle\right) & :=\min _{A B} E^{A B}\left(\left|\psi_{n}\right\rangle\right) \\
& =1-\max _{\left|\phi^{A}\right\rangle\left|\phi^{B}\right\rangle, A B} \mid\left\langle\phi^{A}\left|\left\langle\phi^{B} \mid \psi_{n}\right\rangle\right|^{2}\right. \\
& \equiv 1-\alpha\left(\left|\psi_{n}\right\rangle\right)
\end{aligned}
$$

where the maximum is taken over all pure biseparable states $\left|\phi^{A B}\right\rangle=\left|\phi_{k}^{A}\right\rangle\left|\phi_{n-k}^{B}\right\rangle$ as well as over all possible bipartitions $A B$.

As required by a good measure of entanglement, it can be checked that both bipartite and multipartite entanglement are two non-increasing entanglement measures under LOCCs 22].

Moreover, in order to compute the overlap between a quantum state $\left|\psi_{n}\right\rangle$ and the set of all pure biseparable states with respect to a bipartition $A B$, it is not necessary to explicitly perform the maximization over the whole set: it can be proved [17] that

$$
\alpha^{A B}\left(\left|\psi_{n}\right\rangle\right)=\max _{k=1, \ldots R} s_{k}^{A B}\left(\left|\psi_{n}\right\rangle\right)^{2}
$$

where $\left\{s_{k}^{A B}\left(\left|\psi_{n}\right\rangle\right)\right\}_{k=1}^{R}$ is the set of the Schmidt coefficients of state $\left|\psi_{n}\right\rangle$ with respect to bipartition $A B$ and $R$ is its Schmidt rank.

Definition II.4 (Entanglement witness for genuine multipartite entanglement - [18]). Let $\rho_{\text {ent }}$ be the density matrix representing a completely entangled state of a multipartite quantum system $Q$; let $S_{b i}(Q)$ be the convex set of all states that may be written as a convex combination of biseparable states. Let $W$ be a hermitian operator such that

$$
\left\{\begin{array}{l}
\operatorname{Tr}\left[W \rho_{\text {ent }}\right]<0 \\
\operatorname{Tr}\left[W \rho_{s e p}\right] \geq 0 \forall \rho_{s e p} \in S_{b i}(Q)
\end{array}\right.
$$

Then operator $W$ is an entanglement witness for genuine multipartite entanglement.

A standard procedure for the construction of an entanglement witness that is able to detect genuine multipartite entanglement in the neighbourhood of a given state $|H\rangle$ is that of the projector-based entanglement witness 15 .

$$
W:=\alpha(|H\rangle) \mathbb{I}-|H\rangle\langle H|
$$

where $\alpha(|H\rangle)$ is defined in Eq. (12).

\section{MULTIPARTITE ENTANGLEMENT IN QUANTUM HYPERGRAPH STATES}

In this section we first compute the exact multipartite entanglement formulas for some specific classes of hypergraph states and we then derive a lower bound to the multipartite entanglement of a generic hypergraph state.

We propose a procedure to evaluate exactly the multipartite entanglement for some symmetric classes of hypergraph states. In order to do this we use the concept of infinity norm, defined as follows.

Definition III.1 (Infinity norm). Let $M \in \mathbb{C}^{n \times n}$ be a square matrix. Its infinity norm $\|M\|_{\infty}$ is defined as

$$
\|M\|_{\infty}:=\max _{i=1,2, \ldots n} \sum_{j=1}^{n}\left|M_{i j}\right| .
$$

For $M \geq 0$, its maximum eigenvalue is bounded by $\lambda_{\max }(M) \leq\|M\|_{\infty}($ see for instance [19] $)$.

Our procedure which allows to compute $\alpha^{A B}(|\phi\rangle)$ in Eq. (12) is summarized as follows:

- Take an $n$-qubit hypergraph state $\left|H_{n}\right\rangle$ which is invariant under permutations of the qubits; 
- Consider the bipartition $\bar{A}=\{1,2, \ldots(n-1)\}$ and $\bar{B}=\{n\}$, perform the Schmidt decomposition with respect to this bipartition and spot the maximum Schmidt coefficient $s_{\max }^{\bar{A} \bar{B}}\left(\left|H_{n}\right\rangle\right)$;

- Consider now all other bipartitions $A=\{1,2, \ldots n-$ $k\}$ and $B=\{n-k+1, \ldots n\}$ for $k>1$ and write the reduced density matrix $\rho^{(12 \ldots n-k)}$ corresponding to $n-k$ qubits;

- Compute the infinity norm $\left\|\rho^{(12 \ldots n-k)}\right\|_{\infty}$;

- Compare the infinity norm $\left\|\rho^{(12 \ldots n-k)}\right\|_{\infty}$ with $\left(s_{\max }^{\bar{A} \bar{B}}\left(\left|H_{n}\right\rangle\right)\right)^{2}$

- If $\left\|\rho^{(12 \ldots n-k)}\right\|_{\infty} \leq\left(s_{\max }^{\bar{A} \bar{B}}\left(\left|H_{n}\right\rangle\right)\right)^{2}$ for all values $1<k \leq n / 2$ then $\alpha\left(\left|H_{n}\right\rangle\right)=\left(s_{\max }^{\bar{A} \bar{B}}\left(\left|H_{n}\right\rangle\right)\right)^{2}$. This last step is justified by (13).

In the following we apply this procedure to compute $\alpha\left(\left|H_{n}\right\rangle\right)$, and therefore the multipartite entanglement $E\left(\left|\phi_{n}\right\rangle\right)$ via (12), for some classes of hypergraph states.

\section{A. Hypergraph states with one maximum-cardinality hyperedge}

We here consider $n$-qubit hypergraph states $\left|G_{n}\right\rangle$ with only one maximum-cardinality $n$-hyperedge, namely

$$
\left|G_{n}\right\rangle=C_{n}^{(1,2, \ldots n)}|+\rangle^{\otimes n} .
$$

Theorem III.1 (Multipartite entanglement - One maximum-cardinality hyperedge). Let $\left|G_{n}\right\rangle$ be an n-qubit hypergraph state with just one maximum-cardinality $n$ hyperedge. Then the maximum squared overlap between hypergraph state $\left|G_{n}\right\rangle$ and the pure biseparable states is

$$
\alpha_{n}=\max _{\left|\phi^{A}\right\rangle\left|\phi^{B}\right\rangle,\{A, B\}} \mid\left\langle\phi^{A}\left|\left\langle\phi^{B} \mid G_{n}\right\rangle\right|^{2}=\frac{2^{n-1}-1}{2^{n-1}}\right.
$$

and the multipartite entanglement of hypergraph state $\left|G_{n}\right\rangle$ is

$$
E\left(\left|G_{n}\right\rangle\right)=\frac{1}{2^{n-1}} .
$$

Hypergraph states with only one maximum-cardinality hyperedge are superpositions of all the elements of the computational basis with only one negative sign in front of the element $|11 \ldots 1\rangle$. These are exactly the same states employed by Grover's quantum search algorithm in the single solution case [20]. This result was in fact first proved in Ref. [21], where the entanglement dynamics in Grover's algorithm is analysed. Here we prove it by following the procedure outlined above.
Proof. Consider first the bipartition $\bar{A}=\{1,2, \ldots(n-1)\}$ and $\bar{B}=\{n\}$. The Schmidt decomposition of hypergraph state $\left|G_{n}\right\rangle$ with respect to bipartition $\bar{A} \bar{B}$ is

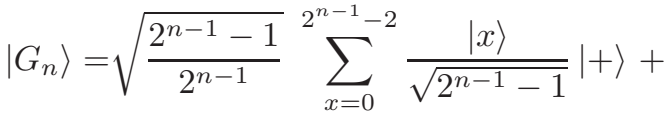

$$
\begin{aligned}
& +\frac{|11 \ldots 1\rangle|-\rangle}{\sqrt{2^{n-1}}}
\end{aligned}
$$

and the maximum Schmidt coefficient is therefore $s_{\max }^{\bar{A} \bar{B}}\left(\left|G_{n}\right\rangle\right)=\sqrt{\frac{2^{n-1}-1}{2^{n-1}}}$.

Consider now bipartitions $A=\{1,2, \ldots n-k\}$ and $B=$ $\{n-k+1, \ldots n\}$ with $k>1$. By performing the partial trace over the last $k$ subsystems, the reduced density matrix $\rho^{(12 \ldots n-k)}$ becomes

$$
\frac{1}{2^{n-1}}\left(\begin{array}{ccccc}
2^{k-1} & 2^{k-1} & \ldots & 2^{k-1} & 2^{k-1}-1 \\
2^{k-1} & 2^{k-1} & \ldots & 2^{k-1} & 2^{k-1}-1 \\
\vdots & \vdots & \ddots & \vdots & \vdots \\
2^{k-1} & 2^{k-1} & \ldots & 2^{k-1} & 2^{k-1}-1 \\
2^{k-1}-1 & 2^{k-1}-1 & \ldots & 2^{k-1}-1 & 2^{k-1}
\end{array}\right)
$$

Regarding the maximum eigenvalue, it follows that

$$
\lambda_{\max }\left(\rho^{(12 \ldots n-k)}\right) \leq\left\|\rho^{(12 \ldots n-k)}\right\|_{\infty}=\frac{2^{n-1}-1}{2^{n-1}}
$$

for all $k>1$. We then conclude that $\alpha_{n}=\frac{2^{n-1}-1}{2^{n-1}}$.

\section{B. Hypergraph states with all (n-1)-hyperedges}

We now consider $n$-qubit hypergraph states $\left|H_{n}^{n-1}\right\rangle$ endowed with all possible hyperedges of cardinality $n-1$, namely

$$
\left|H_{n}^{n-1}\right\rangle=\prod_{i=1}^{n} C_{n-1}^{(1,2, \ldots \hat{i}, \ldots n)}|+\rangle^{\otimes n} .
$$

Theorem III.2 (Multipartite entanglement - Hyperedges of cardinality $n-1)$. Let $\left|H_{n}^{n-1}\right\rangle$ be an n-qubit hypergraph state endowed with all possible hyperedges of cardinality $n-1$. Then the maximum squared overlap between hypergraph state $\left|H_{n}^{n-1}\right\rangle$ and the pure biseparable states is

$$
\left\{\begin{array}{l}
\alpha\left(\left|H_{4}^{3}\right\rangle\right)=\frac{3+\sqrt{5}}{8} \leq \frac{3}{4} \\
\alpha\left(\left|H_{n}^{n-1}\right\rangle\right)=\frac{2^{n-1}-n}{2^{n-1}} \text { for } n \text { even, } n \geq 6 \\
\alpha\left(\left|H_{n}^{n-1}\right\rangle\right)=\frac{2^{n-1}-n+1}{2^{n-1}} \text { for } n \text { odd }
\end{array}\right.
$$


The multipartite entanglement of hypergraph state $\left|H_{n}^{n-1}\right\rangle$ is then given by

$$
\left\{\begin{array}{l}
E\left(\left|H_{4}^{3}\right\rangle\right)=\frac{5-\sqrt{5}}{8} \geq \frac{1}{4} \\
E\left(\left|H_{n}^{n-1}\right\rangle\right)=\frac{n}{2^{n-1}} \text { for } n \text { even, } n \geq 6 \\
E\left(\left|H_{n}^{n-1}\right\rangle\right)=\frac{n-1}{2^{n-1}} \text { for } n \text { odd } .
\end{array}\right.
$$

The complete proof of this result is reported in Appendix $\mathrm{A}$ The procedure is a straightforward generalization of the one applied to the single maximum-cardinality hyperedge. The maximum eigenvalues of the reduced density matrices do not increase for increasing $k$, hence they remain lower than or equal to the squared maximum Schmidt coefficient with respect to the first bipartition $\bar{A} \bar{B}$. The only exception to this behaviour is the case $n=4$, that is the lowest possible even value. We distinguish the case of $n$ even from $n$ odd because of a difference in the sign of the coefficient in front of the computational basis element $|11 \ldots 1\rangle$. While a hypergraph state $\left|H_{n}^{n-1}\right\rangle$ with $n$ even has $n$ negative coefficients, a hypergraph state $\left|H_{n}^{n-1}\right\rangle$ with $n$ odd has an additional negative sign in front of the component $|11 \ldots 1\rangle$ : when $n$ is even the negative signs introduced by the controlled- $Z$ gates compensate each other.

\section{Hypergraph states with all hyperedges of cardinality greater than or equal to $n-1$}

We here consider $n$-qubit hypergraph states endowed with all possible hyperedges of cardinality greater than or equal to $n$ - 1 , namely

$$
\left|H_{n}^{n-1, n}\right\rangle=C_{n}^{(1,2, \ldots n)} \prod_{i=1}^{n} C_{n-1}^{(1,2, \ldots \hat{i}, \ldots n)}|+\rangle^{\otimes n} .
$$

Theorem III.3 (Multipartite entanglement - Hyperedges of cardinality greater than or equal to $n-1)$. Let $\left|H_{n}^{n-1, n}\right\rangle$ be an n-qubit hypergraph state endowed with all possible hyperedges of cardinality greater than or equal to $n-1$. Then the maximum squared overlap between hypergraph state $\left|H_{n}^{n-1, n}\right\rangle$ and the pure biseparable states is

$$
\left\{\begin{array}{l}
\alpha\left(\left|H_{3}^{2,3}\right\rangle\right)=\frac{3}{4} \\
\alpha\left(\left|H_{n}^{n-1, n}\right\rangle\right)=\frac{2^{n-1}-n+1}{2^{n-1}} \text { for } n \text { even } \\
\alpha\left(\left|H_{n}^{n-1, n}\right\rangle\right)=\frac{2^{n-1}-n}{2^{n-1}} \text { for } n \text { odd, } n \geq 5 .
\end{array}\right.
$$

The multipartite entanglement of hypergraph state $\left|H_{n}^{n-1, n}\right\rangle$ is

$$
\left\{\begin{array}{l}
E\left(\left|H_{3}^{2,3}\right\rangle\right)=\frac{1}{4} \\
E\left(\left|H_{n}^{n-1, n}\right\rangle\right)=\frac{n-1}{2^{n-1}} \text { for } n \text { even } \\
E\left(\left|H_{n}^{n-1, n}\right\rangle\right)=\frac{n}{2^{n-1}} \text { for } n \text { odd, } n \geq 5
\end{array}\right.
$$

The complete proof of this result is reported in Appendix $\mathrm{A}$. The procedure is a straightforward generalization of the one applied to the single maximum-cardinality hyperedge case and it is analogous to the procedure applied to prove Theorem III.2, it only differs in the opposite role played by the parity of the number of qubits $n$. The additional $n$-hyperedge, with respect to the previous case, changes the sign of the coefficient in front of the component $|11 \ldots 1\rangle$. We here hence distinguish the case of $n$ even from case of $n$ odd, just like we did in the previous case, perform the same demonstrative procedure and find inverted formulas between the two cases of $n$ even and $n$ odd. As expected, the only exception here is for $n=3$, that is the lowest possible value for $n$ odd, similarly to the previous exception of the case $n=4$ (the lowest possible value for $n$ under the hypothesis of Theorem [II.2).

\section{Lower bound to the multipartite entanglement of a generic hypergraph state}

Theorem III.4 (Multipartite entanglement - General case). Let $\left|H_{n}^{k_{\max }}\right\rangle$ be an n-qubit connected hypergraph state of maximum hyperedge-cardinality equal to $k_{\max }$. Then its overlap with the pure biseparable states is upper bounded by

$$
\alpha\left(\left|H_{n}^{k_{\max }}\right\rangle\right) \leq \frac{2^{k_{\max }-1}-1}{2^{k_{\max }-1}} .
$$

Its multipartite entanglement is hence lower bounded by

$$
E\left(\left|H_{n}^{k_{\max }}\right\rangle\right) \geq \frac{1}{2^{k_{\max }-1}} .
$$

Proof. The proof of the theorem may be outlined as follows.

- Given an $n$-qubit connected hypergraph state $\left|H_{n}^{k_{\max }}\right\rangle$ of maximum hyperedge-cardinality equal to $k_{\max }$, we consider a possible bipartition $A B$. Among the hyperedges that cross the bipartition we choose one with the highest cardinality, which we denote as $\kappa$ : by definition $\kappa \leq k_{\max }$. The reason why we choose an hyperedge with the highest cardinality will be made clear in the next steps.

- We show that hypergraph state $\left|H_{n}^{k_{\max }}\right\rangle$ may always be reduced to a mixture of single-hyperedge hypergraph states $\left|G_{\kappa^{\prime}}\right\rangle$ with $\kappa^{\prime} \leq \kappa \leq k_{\max }$ by only means of operations that are local with respect to the chosen bipartition $A B$.

- Given the non-increasing property of bipartite entanglement under LOCCs, the entanglment of the initial state is greater than or equal to the weighted average of the entanglement values of the single 
states belonging to the mixture, with weights given by the probabilities of the measurement outcomes. In particular the initial entanglement is greater than or equal to the minimum value entering the weighted average. We deduce that

$$
E^{A B}\left(\left|H_{n}^{k_{\max }}\right\rangle\right) \geq E^{A B}\left(\left|G_{\kappa^{\prime}}\right\rangle\right)
$$

where $\kappa^{\prime}$ is the maximum cardinality within the above mixture.

- Recalling that multipartite entanglement is defined as the minimum of bipartite entanglement over all possible bipartitions and applying Theorem III.1. it follows that

$$
E^{A B}\left(\left|H_{n}^{k_{\max }}\right\rangle\right) \geq E\left(\left|G_{\kappa^{\prime}}\right\rangle\right)=\frac{1}{2^{\kappa^{\prime}-1}}
$$

where $E\left(\left|G_{\kappa^{\prime}}\right\rangle\right)$ denotes the minimum of $E^{A B}\left(\left|G_{\kappa^{\prime}}\right\rangle\right)$ over all possible bipartitions AB.

- We conclude by observing that the minimum value of the multipartite entanglement is attained when $\kappa=\kappa^{\prime}=k_{\max }$. In general $\kappa^{\prime} \leq \kappa \leq k_{\max }$ but it is possible that $\kappa^{\prime}=k_{\max }$ if the initially considered bipartition crosses a $k_{\text {max }}$-hyperedge. In general if $\kappa_{1} \leq \kappa_{2}$ then $\frac{1}{2^{\kappa_{1}-1}} \geq \frac{1}{2^{\kappa_{2}-1}}$ and, since we are looking for the minimum, this motivates the choice of the hyperedge with highest cardinality at the first step. This leads to

$$
E\left(\left|H_{n}^{k_{\max }}\right\rangle\right) \geq E\left(\left|G_{k_{\max }}\right\rangle\right)=\frac{1}{2^{k_{\max }-1}} .
$$

In order to complete the proof it hence suffices to show how to reduce $\left|H_{n}^{k_{\max }}\right\rangle$ to a single-hyperedge hypergraph state $\left|G_{\kappa^{\prime}}\right\rangle$ with $\kappa^{\prime} \leq k_{\max }$ by only means of operations that are local with respect to the chosen bipartition $A B$ and single-qubit measurements. This may be achieved through the following iterative procedure (see Fig 1 for an example).

- Given a bipartition $A B$, choose one of the hyperedges with the highest cardinality crossed by the bipartition and call $\kappa$ its cardinality.

- Perform $\sigma_{z}$ measurements on the $n-\kappa$ qubits not belonging to the chosen hyperedge (Fig 11 step 1). The resulting state will be of the form

$$
\left|H_{\kappa}\right\rangle\left|\phi^{(1)}\right\rangle\left|\phi^{(2)}\right\rangle \ldots\left|\phi^{(n-\kappa)}\right\rangle
$$

where $\left|H_{\kappa}\right\rangle$ is a $\kappa$-qubit hypergraph state with an hyperedge with highest cardinality $\kappa$ and possibly other internal lower-cardinality hyperedges; state $\left|\phi^{(i)}\right\rangle \in\{|0\rangle,|1\rangle\}$ is the single-qubit state of the qubit at vertex $i$ and depends on the corresponding measurement output. These measurements do not delete the chosen $\kappa$-hyperedge but may cause the appearance of internal lower-cardinality hyperedges [4].

- Remove all internal hyperedges of cardinality $\kappa-1$ by means of local Pauli operations [14] (Fig,1] step 2). This may introduce edges of lower cardinality that in general may not be removed by only means of LOCCs.

- Remove all hyperedges of cardinality $k^{\prime}<\kappa-1$ that do not cross the chosen bipartition by means of controlled gates of the form $C_{k^{\prime}}$; even if these are not single-qubit transformations they are local with respect to the chosen bipartition. The non-increasing property of bipartite entanglement under LOCCs therefore applies to this case as well.

- Stop if at this stage all lower-cardinality hyperedges have been removed, i.e. the initial state has been reduced to a state of the form $\left|G_{\kappa}\right\rangle\left|\phi^{(1)}\right\rangle\left|\phi^{(2)}\right\rangle \ldots\left|\phi^{(n-\kappa)}\right\rangle$ (Fig 1, step 3 left).

- If this is not the case (Fig[1, step 3 right), it means that the remaining state is still of the form $\left|H_{\kappa}\right\rangle\left|\phi^{(1)}\right\rangle\left|\phi^{(2)}\right\rangle \ldots\left|\phi^{(n-\kappa)}\right\rangle$ where $\left|H_{\kappa}\right\rangle$ is a $\kappa$ qubit hypergraph state with an hyperedge with highest cardinality $\kappa$ and possibly other internal lower-cardinality hyperedges. Consider then the lower-cardinality hyperedges that remain: they all cross the bipartition because those not crossing the bipartition were removed in the previous steps, moreover they are all of cardinality strictly lower than $\kappa-1$. Select an hyperedge with highest cardinality and denote its cardinality with $\tilde{\kappa}$. Measure one of the qubits outside the $\tilde{\kappa}$-hyperedge but still within the $\kappa$-hyperedge; this may cause, depending on the measurement outcome, the appearance of a $(\kappa-1)$-hyperedge crossing the bipartition. Select now again an hyperedge with the highest cardinality among those crossing the bipartition, call $\kappa^{\prime}$ its cardinality, repeat the procedure from the beginning replacing $\kappa$ with $\kappa^{\prime}$.

\section{ENTANGLEMENT WITNESSES IN THE HYPERGRAPH FORMALISM}

In this section we apply the multipartite entanglement results of Section III to the construction of entanglement witnesses. We first derive entanglement witnesses of the projective type, then propose a class of witnesses based on the stabilizer formalism, hence called stabilizer 


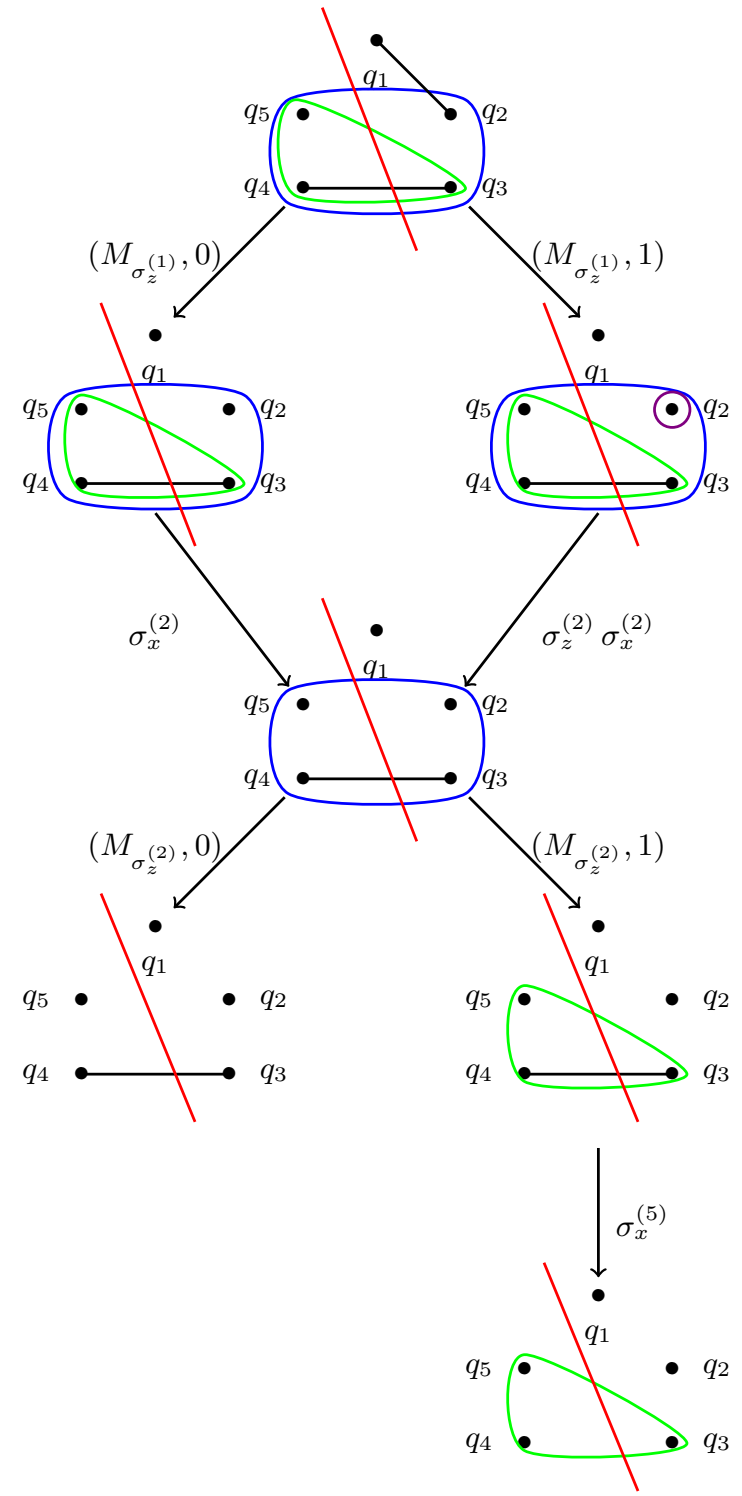

Figure 1. Procedure to transform a 5-qubit hypergraph state of maximum hyperedge cardinality equal to 4 probabilistically into a single-hyperedge hypergraph state by only means of transformations that are local with respect to a chosen bipartition. The exemplifying bipartition is $A=\{1,2,3\}$ and $B=\{4,5\}$ (red inclined line). $\left(M_{\sigma_{z}^{(i)}}, j\right)$ denotes a $\sigma_{z}$ measurement to be performed on qubit $i$ with outcome $j$. Depending on the measurement outcomes the output state may be either of the form $\left|G_{2}\right\rangle\left|\phi^{(1)}\right\rangle\left|\phi^{(2)}\right\rangle\left|\phi^{(5)}\right\rangle$ or of the form $\left|G_{3}\right\rangle\left|\phi^{(1)}\right\rangle\left|\phi^{(2)}\right\rangle$. This leads to a lower bound of $\frac{1}{4}$ for the bipartite entanglement with respect to this choice of the bipartition.

witnesses, requiring a lower number of measurement settings than the projective ones. The efficiency of the constructed witnesses is evaluated on the basis of their robustness to noise and of the number of local measurement settings required by each of them in order to be measured.
Let $W_{n}$ be an entanglement witness able to detect entanglement in the neighbourhood of the $n$-qubit hypergraph state $\left|H_{n}\right\rangle$. Let $R_{p}$ be the hypergraph state $\left|H_{n}\right\rangle$ after the action of some white noise, i.e. $R_{p}=$ $p \frac{\mathbb{I}}{2^{n}}+(1-p)\left|H_{n}\right\rangle\left\langle H_{n}\right|$ with $0 \leq p \leq 1$. We define as robustness parameter the limit value $p_{n}^{L}$ for $p$, such that $\operatorname{Tr}\left[R_{p} W_{n}\right]<0$ for all $p<p_{n}^{L}$. Note that $p_{n}^{L}$ also quantifies the dimensions of neighbourhood where $W_{n}$ is able to detect entanglement.

We remind that a generic witness operator can be decomposed in terms of a set of local observables $\mathcal{O}=$ $\left\{O^{(i)}\right\}_{i=1}^{m \leq n}$, i.e. $O^{(i)}$ acts on qubit $i$ for $i=1,2, \ldots 1, m \leq$ $n$ [23]. We say that we measure the local measurement setting $\mathcal{O}$ if we perform the simultaneous von Neumann measurement of the observables in $\mathcal{O}$. The evaluation of the number of local measurement settings required to measure the expectation value of each witness operator is reported in Appendix B.

\section{A. Projector-based entanglement witnesses}

In the following we list the projector-based entanglement witnesses that we constructed, specifying the value of their associated robustness parameter and the number of local measurement settings required. For the detection of multipartite entanglement in the neighbourhood of the state $\left|G_{n}\right\rangle$, the projector-based witness reads

$$
W_{n}=\frac{2^{n-1}-1}{2^{n-1}} \mathbb{I}-\left|G_{n}\right\rangle\left\langle G_{n}\right|
$$

which needs $\frac{3^{n}-1}{2}$ local measurement settings, see App. $\mathrm{B}$, and has robustness parameter $p_{n}^{L}=\frac{2}{2^{n}-1}$. Because of the maximum-cardinality hyperedge, this case is the worst case scenario regarding the number of measurement settings. All of the following projective witnesses require a number of local measurement settings lower than or equal to (but possibly as high as) $\frac{3^{n}-1}{2}$.

Starting from the state $\left|H_{n}^{n-1}\right\rangle$ we have the projectorbased witnesses

$$
\left\{\begin{array}{l}
W_{4}=\frac{3+\sqrt{5}}{8} \mathbb{I}-\left|H_{4}^{3}\right\rangle\left\langle H_{4}^{3}\right|, \quad n=4 \\
W_{n}=\frac{2^{n-1}-n}{2^{n-1}} \mathbb{I}-\left|H_{n}^{n-1}\right\rangle\left\langle H_{n}^{n-1}\right|, \quad n \geq 6 \text { even } \\
W_{n}=\frac{2^{n-1}-n+1}{2^{n-1}} \mathbb{I}-\left|H_{n}^{n-1}\right\rangle\left\langle H_{n}^{n-1}\right|, \quad n \geq 3 \text { odd } .
\end{array}\right.
$$

with $p_{4}^{L}=\frac{10-2 \sqrt{5}}{15}, p_{n}^{L}=\frac{2 n}{2^{n}-1}$ for $n$ even, $p_{n}^{L}=\frac{2(n-1)}{2^{n}-1}$ for $n$ odd.

The projector-based witnesses for hypergraph states with hyperedges of cardinality $n$ and $n-1$ read 


$$
\left\{\begin{array}{l}
W_{3}=\frac{3}{4} \mathbb{I}-\left|H_{3}^{2,3}\right\rangle\left\langle H_{3}^{2,3}\right|, \quad n=3 \\
W_{n}=\frac{2^{n-1}-n}{2^{n-1}} \mathbb{I}-\left|H_{n}^{n-1, n}\right\rangle\left\langle H_{n}^{n-1, n}\right|, \quad n \geq 5 \text { odd } \\
W_{n}=\frac{2^{n-1}-n+1}{2^{n-1}} \mathbb{I}-\left|H_{n}^{n-1, n}\right\rangle\left\langle H_{n}^{n-1, n}\right|, \quad n \geq 4 \text { even }
\end{array}\right.
$$

with $p_{3}^{L}=\frac{2}{7}, p_{n}^{L}=\frac{2(n-1)}{2^{n}-1}$ for $n$ even, $p_{n}^{L}=\frac{2 n}{2^{n}-1}$ for $n$ odd.

Finally, for a generic hypergraph state with hyperedges of maximum cardinality $k_{\max }$ we have the witness

$$
W_{n}=\frac{2^{k_{\max }-1}-1}{2^{k_{\max }-1}} \mathbb{I}-\left|H_{n}^{k_{\max }}\right\rangle\left\langle H_{n}^{k_{\max }}\right|
$$

with robustness threshold $p_{n}^{L}=\frac{2^{n-k_{\max }+1}}{2^{n}-1}$.

\section{B. Stabilizer entanglement witnesses}

We now construct entanglement witnesses of the form

$$
\tilde{W}_{n}=\beta_{n} \mathbb{I}-\sum_{i=1}^{n} K_{i}
$$

with $\beta_{n} \in \mathbb{R}_{+}$, exploiting the stabilizer formalism and generalizing the procedure proposed in Refs. 24] and [25]. As mentioned above, projector-based entanglement witnesses need a number of local measurement settings that in general is exponentially growing with the number of qubits. The aim of the stabilizer construction is hence to improve this experimental efficiency. The stabilizer entanglement witnesses we propose indeed need a number of local measurement settings that grows linearly with the number of qubits. However, they are less fine that the projector-based ones and display a lower robustness parameter.

In order to determine suitable values for $\beta_{n}$ such that $\tilde{W}_{n}$ is an entanglement witness we require that $\tilde{W}_{n}-$ $C W_{n} \geq 0$ for some positive constant $C>0$. If this holds we have that

$$
\operatorname{Tr}\left[\rho \tilde{W}_{n}\right] \geq C \operatorname{Tr}\left[\rho W_{n}\right]
$$

and $\tilde{W}_{n}$ is still a good entanglement witness. Its robustness parameter is $\frac{n-\beta_{n}}{n}$. In order to maximize $\tilde{p}_{L}^{n}$ we hence need to minimize $\beta_{n}$.

In order to require (36) we compare the two witnesses $W_{n}$ and $\tilde{W}_{n}$ by means of the hypergraph state basis. The action of $W_{n}$ on the hypergraph state basis (4) is

$$
\left\{\begin{array}{l}
W_{n}\left|\phi_{00 \ldots 0}\right\rangle=\alpha\left(\left|H_{n}\right\rangle\right)-1 \\
W_{n}\left|\phi_{x \neq 00 \ldots 0}\right\rangle=\alpha\left(\left|H_{n}\right\rangle\right)
\end{array}\right.
$$

Inequality (36) results in the following set of constraints for the parameter $\beta_{n}$

$$
\left\{\begin{array}{l}
0>\beta_{n}-n \geq C\left(\alpha\left(\left|H_{n}\right\rangle\right)-1\right) \\
\beta_{n}+n \geq C \alpha\left(\left|H_{n}\right\rangle\right) \\
\beta_{n} \pm(n-2) \geq C \alpha\left(\left|H_{n}\right\rangle\right) \\
\cdots \\
\beta_{n} \pm(n-2 m) \geq C \alpha\left(\left|H_{n}\right\rangle\right) \\
\cdots \\
\beta_{n} \geq C \alpha\left(\left|H_{n}\right\rangle\right)
\end{array}\right.
$$

for $n$ even, $m$ positive integer with $0<m<n / 2$ and $C>0$, while

$$
\left\{\begin{array}{l}
0>\beta_{n}-n \geq C\left(\alpha\left(\left|H_{n}\right\rangle\right)-1\right) \\
\beta_{n}+n \geq C \alpha\left(\left|H_{n}\right\rangle\right) \\
\beta_{n} \pm(n-2) \geq C \alpha\left(\left|H_{n}\right\rangle\right) \\
\cdots \\
\beta_{n} \pm(n-2 m) \geq C \alpha\left(\left|H_{n}\right\rangle\right) \\
\cdots \\
\beta_{n} \pm 1 \geq C \alpha\left(\left|H_{n}\right\rangle\right)
\end{array}\right.
$$

for $n$ odd, $m$ and $C$ as above.

These inequalities are all compatible with each other for every $n$ and define a convex compatibility region for $\beta_{n}$ and $C$ where one can minimize $\beta_{n}$ (see Figure 2 for a pictorial representation). The first two inequalities (first line) in the set of constraints are identified by the region below the black line and the one on the right of the blue one, while the inequality in the third line of the set is identified by the region on the left of the green line. Notice that if the latter is satisfied then also all the remaining constraints in the set are automatically satisfied. The optimal value for $\beta_{n}$, given by the minimum compatible with the set of constraints, is at the intersection of the (blue and green) lines

$$
\left\{\begin{array}{l}
\beta_{n}=n-C\left(1-\alpha\left(\left|H_{n}\right\rangle\right)\right) \\
\beta_{n}=(n-2)+C \alpha\left(\left|H_{n}\right\rangle\right)
\end{array}\right.
$$

which corresponds to $C=2$ and $\beta_{n}=n-2\left(1-\alpha\left(\left|H_{n}\right\rangle\right)\right.$.

In the following we list the stabilizer entanglement witnesses constructed in ths way, specifying the value of their associated robustness parameter and the number of local measurement settings required.

A suitable witness for the detection of entanglement in the neighbourhood of $\left|G_{n}\right\rangle$ is given by

$$
\tilde{W}_{n}=\frac{n 2^{n-1}-2}{2^{n-1}} \mathbb{I}-\sum_{i=1}^{n} K_{i}\left(G_{n}\right)
$$

requiring exactly $n$ local measurement settings and with $\tilde{p}_{n}^{L}=\frac{2}{n 2^{n-1}}$. Here $K_{i}\left(G_{n}\right)$ denotes the stabilizer operators for hypergraph state $\left|G_{n}\right\rangle$. Just like in the projective case, this is the worst case scenario regarding the number 


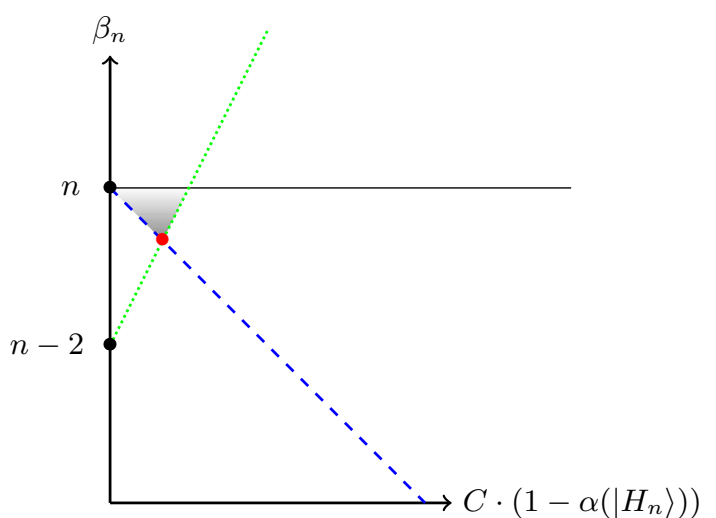

Figure 2. Grey region: feasible region. Black line: $\beta_{n}=n$. Blue dashed line: $\beta_{n}=n-C\left(1-\alpha\left(\left|H_{n}\right\rangle\right)\right)$. Green dotted line: $\beta_{n}=(n-2)+C \alpha\left(\left|H_{n}\right\rangle\right)$. Red dot: optimal value for $\beta_{n}$.

of measurement settings. This means that the number of measurements required by the stabilizer witnesses that follow is limited from above by $n$, hence no more by an exponential function of the number of qubits but by a linear one. A comparison between the robustness parameter for the projective witness (31) and the stabilizer witness (41) is reported in Fig. 3. As we can see, stabilizer entanglement witnesses need fewer local measurement settings than the projective ones but, as a drawback, they are less robust to noise than the projective ones.

Suitable stabilizer witnesses for the detection of entanglement in the neighbourhood of $\left|H_{n}^{n-1}\right\rangle$ are

$$
\left\{\begin{array}{l}
\tilde{W}_{4}=\frac{11+\sqrt{5}}{4} \mathbb{I}-\sum_{i=1}^{4} K_{i}\left(H_{4}^{3}\right), n=4 \\
\tilde{W}_{n}=\frac{n 2^{n-1}-2 n}{2^{n-1}} \mathbb{I}-\sum_{i=1}^{n} K_{i}\left(H_{n}^{n-1}\right), \quad n \geq 6 \text { even }(42) \\
\tilde{W}_{n}=\frac{n 2^{n-1}-2 n+2}{2^{n-1}} \mathbb{I}-\sum_{i=1}^{n} K_{i}\left(H_{n}^{n-1}\right), n \geq 3 \text { odd } .
\end{array}\right.
$$

with $\tilde{p}_{4}^{L}=\frac{5-\sqrt{5}}{16}, \tilde{p}_{n}^{L}=\frac{2}{2^{n-1}}$ for $n$ even, $\tilde{p}_{n}^{L}=\frac{2(n-1)}{n 2^{n-1}}$ for $n$ odd.

For the detection of entanglement in the neighbourhood of $\left|H_{n}^{n-1, n}\right\rangle$ we have

$$
\left\{\begin{array}{l}
\tilde{W}_{3}=\frac{5}{2} \mathbb{I}-\sum_{i=1}^{3} K_{i}\left(H_{3}^{2,3}\right), n=3 \\
\tilde{W}_{n}=\frac{n 2^{n-1}-2 n}{2^{n-1}} \mathbb{I}-\sum_{i=1}^{n} K_{i}\left(H_{n}^{n-1, n}\right), \quad n \geq 5 \text { odd } \\
\tilde{W}_{n}=\frac{n 2^{n-1}-2 n+2}{2^{n-1}} \mathbb{I}-\sum_{i=1}^{n} K_{i}\left(H_{n}^{n-1, n}\right), \quad n \geq 4 \text { even } .
\end{array}\right.
$$

with $\tilde{p}_{3}^{L}=\frac{1}{6}, \tilde{p}_{n}^{L}=\frac{2(n-1)}{n 2^{n-1}}$ for $n$ even, $\tilde{p}_{n}^{L}=\frac{2}{2^{n-1}}$ for $n$ odd.
Suitable stabilizer witnesses for the detection of entanglement in the neighbourhood of a generic hypergraph state with hyperedges with maximum cardinality $k_{\max }$ are given by

$$
\tilde{W}_{n}=\frac{n 2^{k_{\max }-1}-2}{2^{k_{\max }-1}} \mathbb{I}-\sum_{i=1}^{n} K_{i}\left(H_{k_{\max }}\right)
$$

with $\tilde{p}_{n}^{L}=\frac{2}{n 2^{\text {max }-1}}$. A derivation of bounds on the number of measurement settings required to measure the expectation values of the projective witnesses versus the stabilizer ones is reported in Appendix B.

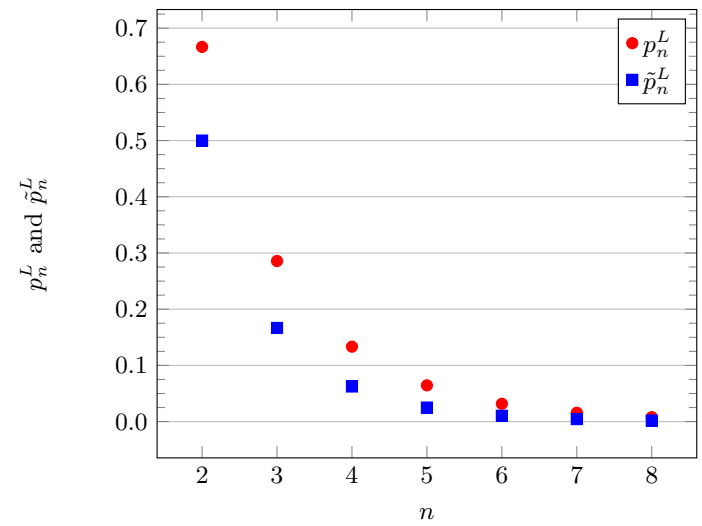

Figure 3. Entanglement detection in the neighbourhood of a $n$-qubit hypergraph state $\left|G_{n}\right\rangle$ with only one maximumcardinality hyperedge. Plot of the robustness parameters $p_{n}^{L}$ (red line with dots) and $\tilde{p}_{n}^{L}$ (blue line with squares) versus the number of qubits $n$ for $2 \leq n \leq 8$.

\section{CONCLUSIONS}

In this work we have studied the entanglement properties of some classes of symmetric hypergraph states for an arbitrary number of qubits $n$ by proposing an analytical procedure based on the notion of infinity norm of a matrix. We have also derived lower bounds to the multipartite entanglement of a generic hypergraph state, that depends on the value of the maximal cardinality of the corresponding hypergraph. We have then constructed two classes of entanglement witness operators for the detection of multipartite entanglement in the neighborhood of a hypergraph state and we have compared their efficiency in terms of minimal number of measurement settings required in order to measure the expectation value of the witness operator and in terms of the corresponding robustness parameter. The analysis has shown that projector-based witnesses perform better in terms of robustness parameter with respect to stabilizer witnesses, but in general they are more demanding in terms of the number of measurement settings required. 


\section{ACKNOWLEDGMENTS}

We thank O. Gühne and M. Gachechiladze for suggestions to improve a previous version of this manuscript. DB acknowledges support by BMBF.

\section{Appendix A: Proofs of the multipartite entanglement formulas}

It suffices here to prove the overlap and multipartite entanglement formulas of Theorem III.2. The proof of Theorem III.3 is based on the same procedure, with the only difference being in the role played by the parity of the number of qubits and the exception of case $n=3$.

Proof. Case $n$ even. We consider the representation of hypergraph state $\left|H_{n}^{n-1}\right\rangle$ over the computational basis and note that the number of negative signs in front of the computational basis elements is equal to $\left(\begin{array}{c}n \\ n-1\end{array}\right) \equiv n$, while the coefficient of state $|11 \ldots 1\rangle$ is equal to +1 . The Schmidt decomposition of hypergraph state $\left|H_{n}^{n-1}\right\rangle$ with respect to bipartition $\bar{A}=\{1,2, \ldots(n-1)\}$ and $\bar{B}=\{n\}$ may always be written as

$$
\begin{aligned}
\left|H_{n}^{n-1}\right\rangle & =\sqrt{\frac{2^{n-1}-n}{2^{n-1}}} \sum_{\substack{x=0, w(x)<n-2}}^{2^{n-1}-1} \frac{|x\rangle}{\sqrt{2^{n-1}-n}}|+\rangle+ \\
& +\sqrt{\frac{n}{2^{n-1}}} \cdot \frac{1}{\sqrt{n}}\left(\sum_{\substack{x=0, w(x)=n-2}}^{2^{n-1}-1}|x\rangle-|11 \ldots 1\rangle\right)|-\rangle
\end{aligned}
$$

where the first summation is taken over all binary numbers from 0 to $2^{n-1}-1$ with weight $w(x)<n-2$ while the second summation is taken over those binary numbers $x$ that have weight $w(x)$ exactly equal to $n-2$; while the first summation is made up of $2^{n-1}-n$ addends, the second one is made up of $n-1$ terms.

As a straightforward consequence of representation (A1), it follows that the maximum Schmidt coefficient of hypergraph state $\left|H_{n}^{n-1}\right\rangle$, with respect to bipartition $\bar{A} \bar{B}$ and for $n \geq 4$, is $s_{\max }^{\bar{A} \bar{B}}\left(\left|H_{n}^{n-1}\right\rangle\right)=\sqrt{\frac{2^{n-1}-n}{2^{n-1}}}$.

Case $n=4$. We first consider the case with $n=4$ and write hypergraph state $\left|H_{4}^{3}\right\rangle$ as

$$
\begin{aligned}
\left|H_{4}^{3}\right\rangle & =\frac{1}{\sqrt{2}}\left(\frac{|000\rangle+|001\rangle+|010\rangle+|100\rangle}{2}|+\rangle+\right. \\
& \left.+\frac{|011\rangle+|101\rangle+|110\rangle-|111\rangle}{2}|-\rangle\right) .
\end{aligned}
$$

The maximum Schmidt coefficient with respect to bipartition $A=\{1,2,3\}$ and $B=\{4\}$ is $s_{\max }^{A B}\left(\left|H_{4}^{3}\right\rangle\right)=\frac{1}{\sqrt{2}}$, hence the maximum eigenvalue of the corresponding reduced density matrix $\rho^{(123)}$ is $\lambda_{\max }\left(\rho^{(123)}\right)=\frac{1}{2}$.
Taking then into consideration bipartition $A=\{1,2\}$ and $B=\{3,4\}$, we write hypergraph state $\left|H_{4}^{3}\right\rangle$ as

$$
\begin{aligned}
\left|H_{4}^{3}\right\rangle & =\frac{|0\rangle\left|G_{3}\right\rangle+|1\rangle C_{3}^{(2,3,4)}\left|H_{3}^{2}\right\rangle}{\sqrt{2}} \\
& =\frac{1}{2}\left(|00\rangle|+\rangle^{\otimes 2}+(|01\rangle+|10\rangle)\left|G_{2}\right\rangle+|11\rangle\left|H_{2}^{1}\right\rangle\right) .
\end{aligned}
$$

If we now partially trace over both systems 3 and 4, the resulting density matrix is given by

$$
\rho^{(12)}=\frac{1}{16}\left(\begin{array}{cccc}
4 & 2 & 2 & 0 \\
2 & 4 & 4 & -2 \\
2 & 4 & 4 & -2 \\
0 & -2 & -2 & 4
\end{array}\right)
$$

hence $\lambda_{\max }\left(\rho^{(12)}\right) \leq\left\|\rho^{(12)}\right\|_{\infty}=\frac{3}{4}$. We conclude that $\alpha\left(\left|H_{4}^{3}\right\rangle\right) \leq \frac{3}{4}$.

If we explicitly compute the eigenvalues of the matrix $\rho^{(12)}$ it turns out that its maximum eigenvalue is $\lambda_{\max }\left(\rho^{(12)}\right)=\frac{3+\sqrt{5}}{8} \leq \frac{3}{4}$, as expected.

Case $n \geq 6$. Let $\left|H_{n}^{n-1}\right\rangle$ be a $n$-qubit hypergraph state with $n$ even, $n \geq 6$, and with all possible $(n-1)$ hyperedges. Besides the representation (A1), the hypergraph state $\left|H_{n}^{n-1}\right\rangle$ may be written in the following ways

$$
\begin{aligned}
\left|H_{n}^{n-1}\right\rangle & =\frac{|0\rangle\left|G_{n-1}\right\rangle+|1\rangle\left|\tilde{H}_{n-1}^{n-2}\right\rangle}{\sqrt{2}} \\
& =\frac{|00\rangle|+\rangle^{\otimes n-2}+(|01\rangle+|10\rangle)\left|G_{n-2}\right\rangle+|11\rangle\left|\tilde{H}_{n-2}^{n-3}\right\rangle}{2} \\
& =\frac{1}{2 \sqrt{2}}\left((|000\rangle+|001\rangle+|010\rangle+|100\rangle)|+\rangle{ }^{\otimes n-3}+\right. \\
& \left.+(|011\rangle+|101\rangle+|110\rangle)\left|G_{n-3}\right\rangle+|111\rangle\left|\tilde{H}_{n-3}^{n-4}\right\rangle\right) \\
& =\frac{1}{\sqrt{2^{k}}}\left(\sum_{\substack{x=0, w(x)<k-1}}^{2^{k}-1}|x\rangle|+\rangle{ }^{\otimes n-k}+\right. \\
& +\sum_{\substack{x=0, 2^{k}-1}}^{\left.|x\rangle\left|G_{n-k}\right\rangle+|\overbrace{11 \ldots 1}^{k}\rangle\left|\tilde{H}_{n-k}^{n-k-1}\right\rangle\right)}
\end{aligned}
$$

for all $1 \leq k \leq n$, where $\left|\tilde{H}_{n-k}^{n-k-1}\right\rangle$ is the $(n-k)$-qubit hypergraph state with all possible $(n-k-1)$-hyperedges plus an additional $n$-hyperedge if $n-k$ is odd, so that the coefficient in front of $|11 \ldots 1\rangle$ remains equal to +1 for every $k$. While the first summation is made up of $2^{k}-k-1$ terms, the second summation is made up of $k$ addends. As a consequence, we may express the corresponding reduced density matrix $\rho^{(n-k)}$ as

$$
\rho^{(n-k)}=\frac{1}{\mathcal{N}}\left(2^{k}-k-1\right) \mathcal{I}_{2^{n-k}}+\frac{1}{\mathcal{N}} k \tilde{\mathcal{I}}_{2^{n-k}}+\frac{1}{\mathcal{N}} \mathcal{R}_{2^{n-k}}
$$

where $\mathcal{I}_{2^{n-k}} \propto(|+\rangle\langle+|)^{\otimes n-k}$ is the $2^{n-k} \times 2^{n-k}$ matrix filled with ones, $\tilde{\mathcal{I}}_{2^{n-k}} \propto\left|G_{n-k}\right\rangle\left\langle G_{n-k}\right|$ is the $2^{n-k} \times 2^{n-k}$ 
matrix filled with ones except for the last column and for the last row, that have all elements equal to -1 and a final 1, i.e.

$$
\tilde{\mathcal{I}}_{2^{n-k}}=\left(\begin{array}{ccccc}
1 & 1 & \ldots & 1 & -1 \\
1 & 1 & \ldots & 1 & -1 \\
\vdots & \vdots & \ddots & \vdots & \vdots \\
1 & 1 & \ldots & 1 & -1 \\
-1 & -1 & \ldots & -1 & 1
\end{array}\right)
$$

$\mathcal{R}_{2^{n-k}} \propto\left|\tilde{H}_{n-k}^{n-k-1}\right\rangle\left\langle\tilde{H}_{n-k}^{n-k-1}\right|$ and $\mathcal{N}$ is a normalization factor. The matrix $\mathcal{R}_{2^{n-k}}$ has two kinds of rows: the first one has $n-k$ elements equal to -1 and $2^{n-k}-n+k$ elements equal to 1 , in particular the last element is equal to 1 ; the second one has the same elements with opposite sign. The sum of these three matrices gives a matrix whose elements may assume the following 4 possible values

$$
\begin{aligned}
& v_{1}=2^{k}-k-1+k+1=2^{k} \\
& v_{2}=2^{k}-k-1+k-1=2^{k}-2 \\
& v_{3}=2^{k}-k-1-k+1=2^{k}-2 k \\
& v_{4}=2^{k}-k-1-k-1=2^{k}-2 k-2
\end{aligned}
$$

where $v_{1}$ and $v_{2}$ are internal values while $v_{3}$ and $v_{4}$ are values that may be assumed by elements of the last column and row. Values $v_{i}$ for $i=1,2,3,4$ are all nonnegative except for $v_{4}=-2$ when $k=2$.

We can now evaluate $\left\|\rho^{n-k}\right\|_{\infty}$ as

$$
\left\|\rho^{n-k}\right\|_{\infty}=\max \left\{\mathcal{N}_{\infty}^{1}, \mathcal{N}_{\infty}^{2}\right\}
$$

where $\mathcal{N}_{\infty}^{1}$ gathers contributions coming from the first row while $\mathcal{N}_{\infty}^{2}$ has contributions coming from the other possible kind of row.

We will first consider $k=2$. In this case it turns out that $v_{1}=4, v_{2}=2, v_{3}=0$ and $v_{4}=-2$. This means that $\mathcal{N}_{\infty}^{1}$ and $\mathcal{N}_{\infty}^{2}$ take the following values

$$
\begin{gathered}
\mathcal{N}_{\infty}^{1} \stackrel{k=2}{=} \frac{2^{n-1}-n}{2^{n-1}} \\
\mathcal{N}_{\infty}^{2} \stackrel{k=2}{=} \frac{2^{n-1}-n}{2^{n-1}}-\left(\frac{2^{n-2}-2 n+2}{2^{n-1}}\right)
\end{gathered}
$$

where $\frac{2^{n-2}-2 n+2}{2^{n-1}} \geq 0$ for $n \geq 5$. This means that, when $k=2, \mathcal{N}_{\infty}^{1} \geq \mathcal{N}_{\infty}^{2}$ for every $n \geq 6$, with the only exception of the case $n=4$ that has already been examined above. Actually when $n=4$ and $k=2$, we have that $\frac{2^{n-1}-n}{2^{n-1}}=\frac{1}{2}$ and $\frac{2^{n-2}-2 n+2}{2^{n-1}}=-\frac{1}{4}$, so the sum of their absolute values is $\frac{3}{4}$ as expected.

Consider now $k \geq 3$. In this case we have that $\mathcal{N}_{\infty}^{1}$ and $\mathcal{N}_{\infty}^{2}$ may be written as

$$
\begin{aligned}
\mathcal{N}_{\infty}^{1} & =\frac{v_{1}\left(2^{n-k}-n+k-1\right)+v_{2}(n-k)+v_{3}}{\mathcal{N}} \\
& =\frac{2^{n-1}-n}{2^{n-1}}
\end{aligned}
$$

$$
\begin{aligned}
\mathcal{N}_{\infty}^{2} & =\frac{v_{2}\left(2^{n-k}-n+k-1\right)+v_{1}(n-k)+v_{4}}{\mathcal{N}} \\
& =\frac{2^{n-1}-n}{2^{n-1}}-\left(\frac{2^{n-k}-2(n-k)}{2^{n-1}}\right)
\end{aligned}
$$

where $\frac{2^{n-k}-2(n-k)}{2^{n-1}} \geq 0$ for $n-k \geq 2$. Since we need to consider only inequivalent bipartitions, that means $k \leq \frac{n}{2}$, the cases corresponding to $n \geq 6$ and $3 \leq k \leq \frac{n}{2}$ satisfy this requirement. Hence we have that $\mathcal{N}_{\infty}^{1} \geq \overline{\mathcal{N}}_{\infty}^{2}$ for all values of $n \geq 6$ and $3 \leq k \leq \frac{n}{2}$.

We conclude by observing that the value of $\mathcal{N}_{\infty}^{1}$ is independent of $k$ and equal to the squared maximum Schmidt coefficient $s_{\max }^{\bar{A} \bar{B}}\left(\left|H_{n-1}^{n}\right\rangle\right)^{2}$, evaluated with respect to bipartition $\bar{A} \bar{B}$.

Case $n$ odd. We consider the representation of hypergraph state $\left|H_{n}^{n-1}\right\rangle$ over the computational basis and note that the number of negative signs in front of the computational basis elements is equal to $\left(\begin{array}{c}n \\ n-1\end{array}\right)+1 \equiv n+1$, in particular the coefficient of state $|11 \ldots 1\rangle$ is equal to -1 . The Schmidt decomposition of the hypergraph state $\left|H_{n}^{n-1}\right\rangle$ with respect to bipartition $\bar{A} \bar{B}$ may always be written as

$$
\begin{aligned}
\left|H_{n}^{n-1}\right\rangle & =\sqrt{\frac{2^{n-1}-n+1}{2^{n-1}}} \cdot \frac{1}{\sqrt{2^{n-1}-n+1}} \cdot \\
& \cdot\left(\sum_{\substack{x=0, w(x)<n-2}}^{2^{n-1}-1}|x\rangle-|11 \ldots 1\rangle\right)|+\rangle+ \\
& +\sqrt{\frac{n-1}{2^{n-1}}} \sum_{\substack{x=0, w(x)=n-2}}^{2^{n-1}-1} \frac{|x\rangle}{\sqrt{n-1}}|-\rangle
\end{aligned}
$$

where the first summation is taken over all binary numbers ranging from 0 to $2^{n-1}-1$ with weight $w(x)<n-2$ while the second summation is taken over those binary numbers $x$ that have weight $w(x)$ exactly equal to $n-2$; while the first summation is made up of $2^{n-1}-n$ addends, the second one is made up of $n-1$ terms.

As a straightforward consequence of representation (A8), it follows that the maximum Schmidt coefficient of hypergraph state $\left|H_{n}^{n-1}\right\rangle$, with respect to bipartition $\bar{A} \bar{B}$, is $s_{\max }^{\bar{A} \bar{B}}\left(\left|H_{n}^{n-1}\right\rangle\right)=\sqrt{\frac{2^{n-1}-n+1}{2^{n-1}}}$.

Case $n=3$. We first consider case $n=3$ and write the hypergraph state $\left|H_{3}^{2}\right\rangle$ as

$$
\left|H_{3}^{2}\right\rangle=\frac{1}{\sqrt{2}} \frac{|00\rangle-|11\rangle}{\sqrt{2}}|+\rangle+\frac{1}{\sqrt{2}} \frac{|01\rangle+|10\rangle}{\sqrt{2}}|-\rangle .
$$

The maximum Schmidt coefficient with respect to bipartition $A=\{1,2\}$ and $B=\{3\}$ is $s_{\max }^{A B}\left(\left|H_{3}^{2}\right\rangle\right)=\frac{1}{\sqrt{2}}$, hence the maximum eigenvalue of the corresponding reduced density matrix $\rho^{(12)}$ is $\lambda_{\max }\left(\rho^{(12)}\right)=\frac{1}{2}$ and the maximum overlap is $\alpha\left(\left|H_{3}^{2}\right\rangle\right)=\frac{1}{2}$. We do not need to consider any other bipartitions since, given the invariance of the state under permutations of the qubits, they are all equivalent to this one. 
Case $n \geq 5$. Let $\left|H_{n}^{n-1}\right\rangle$ be a $n$-qubit hypergraph state with $n$ odd, $n \geq 5$, and with all possible $(n-1)$ hyperedges. Besides the representation (A8), the hypergraph state $\left|H_{n}^{n-1}\right\rangle$ may be written as

$$
\begin{aligned}
\left|H_{n}^{n-1}\right\rangle & =\frac{|0\rangle\left|G_{n-1}\right\rangle+|1\rangle\left|\hat{H}_{n-1}^{n-2}\right\rangle}{\sqrt{2}} \\
& =\frac{|00\rangle|+\rangle^{\otimes n-2}+(|01\rangle+|10\rangle)\left|G_{n-2}\right\rangle+|11\rangle\left|\hat{H}_{n-2}^{n-3}\right\rangle}{2} \\
& =\frac{1}{2 \sqrt{2}}\left((|000\rangle+|001\rangle+|010\rangle+|100\rangle)|+\rangle^{\otimes n-3}+\right. \\
& \left.+(|011\rangle+|101\rangle+|110\rangle)\left|G_{n-3}\right\rangle+|111\rangle\left|\hat{H}_{n-3}^{n-4}\right\rangle\right) \\
& =\frac{1}{\sqrt{2^{k}}}\left(\sum_{\substack{x=0, w(x)<k-1}}^{2^{k}-1}|x\rangle|+\rangle^{\otimes n-k}+\sum_{\substack{x=0, w(x)=k-1}}^{2^{k}-1}|x\rangle\left|G_{n-k}\right\rangle+\right. \\
& \left.\left.+|\overbrace{11 \ldots 1}^{k}| \hat{H}_{n-k}^{n-k-1}\right\rangle\right)
\end{aligned}
$$

for all $1 \leq k \leq n$, where $\left|\hat{H}_{n-k}^{n-k-1}\right\rangle$ is the $(n-k)$-qubit hypergraph state with all possible $(n-k-1)$-hyperedges plus an additional $n$-hyperedge if $n-k$ is even, so that the coefficient in front of $|11 \ldots 1\rangle$ remains equal to -1 for every $k$. While the first summation is made up of $2^{k}-k-$ 1 terms, the second summation is made up of $k$ addends. As a consequence, we may represent the corresponding reduced density matrix $\rho^{(n-k)}$ as

$$
\rho^{(n-k)}=\frac{1}{\mathcal{N}}\left(2^{k}-k-1\right) \mathcal{I}_{2^{n-k}}+\frac{1}{\mathcal{N}} k \tilde{\mathcal{I}}_{2^{n-k}}+\frac{1}{\mathcal{N}} \mathcal{R}_{2^{n-k}}
$$

where $\mathcal{R}_{2^{n-k}} \propto\left|\hat{H}_{n-k}^{n-k-1}\right\rangle\left\langle\hat{H}_{n-k}^{n-k-1}\right|$ and $\mathcal{N}$ is a normalization factor. The matrix $\mathcal{R}_{2^{n-k}}$ has two kinds of rows: the first one has $n-k+1$ elements equal to -1 and $2^{n-k}-n+k-1$ elements equal to 1 , in particular the last element is equal to -1 ; the second one has the same elements with opposite sign. The sum of these three matrices gives a matrix whose elements may assume the following 4 possible values

$$
\begin{aligned}
& v_{1}=2^{k}-k-1+k+1=2^{k} \\
& v_{2}=2^{k}-k-1+k-1=2^{k}-2 \\
& v_{3}=2^{k}-k-1-k-1=2^{k}-2 k-2 \\
& v_{4}=2^{k}-k-1-k+1=2^{k}-2 k
\end{aligned}
$$

where $v_{1}$ and $v_{2}$ are internal values while $v_{3}$ and $v_{4}$ are values that may be assumed by elements of the last column and row. Values $v_{i}$ for $i=1,2,3,4$ are all nonnegative except for $v_{3}=-2$ when $k=2$.

We can now evaluate $\left\|\rho^{n-k}\right\|_{\infty}$ as

$$
\left\|\rho^{n-k}\right\|_{\infty}=\max \left\{\mathcal{N}_{\infty}^{1}, \mathcal{N}_{\infty}^{2}\right\}
$$

where $\mathcal{N}_{\infty}^{1}$ gathers contributions coming from the first row while $\mathcal{N}_{\infty}^{2}$ has contributions coming from the other possible kind of row.

We consider first $k=2$. In this case it turns out that $v_{1}=4, v_{2}=2, v_{3}=-2$ and $v_{4}=0$. This means that $\mathcal{N}_{\infty}^{1}$ and $\mathcal{N}_{\infty}^{2}$ take the following values

$$
\begin{gathered}
\mathcal{N}_{\infty}^{1} \stackrel{k=2}{=} \frac{2^{n-1}-n+1}{2^{n-1}} \\
\mathcal{N}_{\infty}^{2} \stackrel{k=2}{=} \frac{2^{n-1}-n+1}{2^{n-1}}-\left(\frac{2^{n-3}-n+2}{2^{n-2}}\right)
\end{gathered}
$$

where $\frac{2^{n-3}-n+2}{2^{n-2}} \geq 0$ for $n \geq 3$. This means that, when $k=2$, we have that $\mathcal{N}_{\infty}^{1} \geq \mathcal{N}_{\infty}^{2}$ for every $n \geq 5$.

Consider now $k \geq 3$. In this case we need to consider values of $n$ such that $n \geq 7$. We then find that $\mathcal{N}_{\infty}^{1}$ and $\mathcal{N}_{\infty}^{2}$ may be written as

$$
\begin{aligned}
\mathcal{N}_{\infty}^{1} & =\frac{v_{1}\left(2^{n-k}-n+k-1\right)+v_{2}(n-k)+v_{3}}{\mathcal{N}} \\
& =\frac{2^{n-1}-n-1}{2^{n-1}} \\
& \leq \frac{2^{n-1}-n+1}{2^{n-1}} \\
\mathcal{N}_{\infty}^{2} & =\frac{v_{2}\left(2^{n-k}-n+k-1\right)+v_{1}(n-k)+v_{4}}{\mathcal{N}} \\
& =\frac{2^{n-1}-n-1}{2^{n-1}}-\left(\frac{2^{n-k}-2(n-k)-2}{2^{n-1}}\right)
\end{aligned}
$$

where $\frac{2^{n-k}-2(n-k)}{2^{n-1}} \geq 0$ for $n-k \geq 3$. Since we need to consider only inequivalent bipartitions, that means $k \leq$ $\frac{n-1}{2}$, the cases corresponding to $n \geq 7$ and $3 \leq k \leq \frac{n-1}{2}$ satisfy this requirement. Hence we have that $\mathcal{N}_{\infty}^{1} \geq \mathcal{N}_{\infty}^{2}$ also for all values of $n \geq 7$ and $3 \leq k \leq \frac{n-1}{2}$.

We conclude by observing that the value of $\mathcal{N}_{\infty}^{1}$ is independent of $k$ and it is lower than the squared maximum Schmidt coefficient $s_{\max }^{\bar{A} \bar{B}}\left(\left|H_{n}^{n-1}\right\rangle\right)^{2}$, evaluated with respect to bipartition $\bar{A} \bar{B}$.

\section{Appendix B: Measurement of the witnesses}

a. Single maximum-cardinality hyperedge case In order to measure each stabilizer operator, one local measurement setting is required and they are all different from each other. To be more precise, stabilizer $K_{i}$ is given by the tensor product of the Pauli matrix $\sigma_{x}^{(i)}$ and the control gate $C_{n-1}^{(1,2, \ldots \hat{i}, \ldots n)}$, whose decomposition over the Pauli basis has all possible tensor products of Pauli matrices $\sigma_{z}^{(j)}$ and identities $\mathbb{I}^{(k)}$ for $j, k=1,2, \ldots \hat{i}, \ldots n$. Consequently, the measurement setting required to measure the expectation value of $K_{i}$ is composed of $n-1$ local measurements of type $Z$ and one measurement of kind $X$ to be performed on qubit $i$. 
Consider now compositions of pairs of stabilizers, i.e. $K_{i} K_{j}$. Pauli matrices of type $\sigma_{y}$ appear in positions $i$ and $j$ because of terms $\sigma_{x}^{(i)} \sigma_{z}^{(i)}=-i \sigma_{y}^{(i)}$ and $\sigma_{z}^{(j)} \sigma_{x}^{(j)}=i \sigma_{y}^{(j)}$. Given the hermiticity of the stabilizer operators and of their compositions, among all possible arising new terms, i.e. $\sigma_{y}^{(i)} \otimes \sigma_{y}^{(j)}, \sigma_{x}^{(i)} \otimes \sigma_{x}^{(j)}, i \sigma_{x}^{(i)} \otimes \sigma_{y}^{(j)}$ and $i \sigma_{y}^{(i)} \otimes \sigma_{x}^{(j)}$, only the first two appear. We notice that, due to the hermiticity requirement, only an even number of local operators of kind $Y$ appear. It follows that, in order to measure each composition of pair of stabilizers, two measurement settings are required: one with measurements of kind $X$ and one with measurements of kind $Y$ to be performed on qubits $i$ and $j$.

We consider now the composition of the pair $K_{i} K_{j}$ with stabilizer $K_{k}$. We have to consider first the new terms arising from the composition of $\sigma_{x}^{(i)} \otimes \sigma_{x}^{(j)} \otimes \sigma_{z}^{(k)}$ and $\sigma_{x}^{(i)} \otimes \sigma_{x}^{(j)} \otimes \mathbb{I}^{(k)}$ with $\sigma_{z}^{(i)} \otimes \sigma_{z}^{(j)} \otimes \sigma_{x}^{(k)}, \mathbb{I}^{(i)} \otimes \mathbb{I}^{(j)} \otimes \sigma_{x}^{(k)}$, $\sigma_{z}^{(i)} \otimes \mathbb{I}^{(j)} \otimes \sigma_{x}^{(k)}$ and $\mathbb{I}^{(i)} \otimes \sigma_{z}^{(j)} \otimes \sigma_{x}^{(k)}$; the new terms are 8 but, because of the hermiticity requirement, only half of them appear. We then consider those terms arising from the composition of $\sigma_{y}^{(i)} \otimes \sigma_{y}^{(j)} \otimes \sigma_{z}^{(k)}$ and $\sigma_{y}^{(i)} \otimes \sigma_{y}^{(j)} \otimes \mathbb{I}^{(k)}$ with $\sigma_{z}^{(i)} \otimes \sigma_{z}^{(j)} \otimes \sigma_{x}^{(k)}, \mathbb{I}^{(i)} \otimes \mathbb{I}^{(j)} \otimes \sigma_{x}^{(k)}, \sigma_{z}^{(i)} \otimes \mathbb{I}^{(j)} \otimes \sigma_{x}^{(k)}$ and $\mathbb{I}^{(i)} \otimes \sigma_{z}^{(j)} \otimes \sigma_{x}^{(k)}$; they are 8 but, because of the hermiticity requirement, only half of them appear. The 4 terms that survive in the first round are just the same as those that survive in the second; actually, the four terms generated in each round alone exhaust all admissible terms. In order to measure the operator $K_{i} K_{j} K_{k}$, we conclude that 4 local measurement settings are therefore required. In fact, due to the hermiticity requirement, only an even number of local measurements of kind $Y$ needs to be performed: the number of ways in which we can choose an even set of qubits among 3 , onto which perform local measurements of kind $Y$, is indeed exactly equal to 4 .

This reasoning may be extended to any compositions of stabilizer operators of the form $\prod_{i=1}^{k} K_{i}$ with $2 \leq k \leq$ $n$. Because of the hermiticity requirement, only an even number of local operators of kind $Y$ appears: the number of ways in which we can choose an even set of qubits among $k$, onto which perform local measurements of kind $Y$, is equal to $n_{k}$ where

$$
n_{k}=\sum_{k^{\prime}=0, \text { even }}^{k}\left(\begin{array}{c}
k \\
k^{\prime}
\end{array}\right) \equiv 2^{k-1} .
$$

It follows that $2^{k-1}$ is the number of local measurement settings required to measure the expectation value of the product of $k$ stabilizer [26]. Moreover, regarding the number of local measurement settings required, the sole maximum-cardinality hyperedge case is the most demanding: the local decomposition of the control gate $C_{n-1}^{(1,2, \ldots \hat{i}, \ldots n)}$ has all possible tensor products of Pauli matrices $\sigma_{z}^{(j)}$ and identities $\mathbb{I}^{(k)}$ for $j, k=1,2, \ldots \hat{i}, \ldots n$.
This happens to the stabilizer operators of any hypergraph states endowed with a maximum-cardinality hyperedge. Any other $k$-qubit control gate with $k<n-1$ has in its decomposition a subset of the terms appearing in the representation of control gate $C_{n-1}^{(1,2, \ldots \hat{i}, \ldots n)}$.

We want to point out that the vanishing of antihermitian terms and the survival of the hermitian ones may be explained also in the following way. Consider the composition of stabilizers $K_{i}$ and $K_{j}$, then an odd number of $\sigma_{y}$ matrices may appear as a consequence either of composition $\sigma_{x}^{(i)} \sigma_{z}^{(i)}=-i \sigma_{y}^{(i)}$ in position $i$ or of composition $\sigma_{z}^{(j)} \sigma_{x}^{(j)}=i \sigma_{y}^{(j)}$ in position $j$. Because of the hermiticity requirement, terms $i \sigma_{y}^{(i)}$ and $-i \sigma_{y}^{(j)}$ should appear too. Terms with an even number of $\sigma_{y}$ matrices arise from the following tensor products: $i \sigma_{y}^{(i)} \otimes(-i) \sigma_{y}^{(j)}=\sigma_{y}^{(i)} \otimes \sigma_{y}^{(j)}$ and $-i \sigma_{y}^{(i)} \otimes i \sigma_{y}^{(j)}=\sigma_{y}^{(i)} \otimes \sigma_{y}^{(j)}$. This means that in the even case, despite the single $\sigma_{y}$ matrices having opposite signs, their tensor product results in having the same sign.

As regards the projective witness $W_{n}$, we express the projector $\left|G_{n}\right\rangle\left\langle G_{n}\right|$ as the sum of the identity plus $n$ other different contributions, each contribution made up of terms of the form $\prod_{i=1}^{j} K_{i}$ for $j=1,2, \ldots n$, i.e.

$$
\begin{aligned}
W_{n} & =\frac{2^{n-1}-1}{2^{n-1}} \mathbb{I}-\left|G_{n}\right\rangle\left\langle G_{n}\right| \\
& =\frac{2^{n-1}-1}{2^{n-1}} \mathbb{I}-\prod_{i=1}^{n} \frac{K_{i}+\mathbb{I}}{2} \\
& =\frac{2^{n-1}-1}{2^{n-1}} \mathbb{I}+ \\
& -\frac{1}{2^{n}}\left(\mathbb{I}+\sum_{i=1}^{n} K_{i}+\sum_{i<j=1}^{n} K_{i} K_{j}+\ldots \prod_{i=1}^{n} K_{i}\right) .
\end{aligned}
$$

Then the number of local measurement settings required by the witness $W_{n}$ is $\sum_{k=1}^{n} 2^{k-1}\left(\begin{array}{l}n \\ k\end{array}\right)=\frac{3^{n}-1}{2}$. The witness $\tilde{W}_{n}$ instead, since it can be written as a sum of single stabilizers, needs exactly $n$ local measurement settings in order to be measured.

We conclude that, on the one hand, witness $\tilde{W}_{n}$ may always be measured efficiently: the number of local measurement settings required grows linearly with $n$. On the other hand, the number of local measurement settings required by the projective witness $W_{n}$, is not only strictly greater than the number required by the corresponding stabilizer witness $\tilde{W}_{n}$, but, in the worst case scenario, grows exponentially with the number of qubits $n$.

b. General case In order to measure each stabilizer operator, one local measurement setting is needed but it may happen that some stabilizers require the same local measurement setting. Let for instance $K_{i}$ and $K_{j}$ be the stabilizer operators associated to a pair of qubits $i$ 
and $j$ not directly connected to each other. Then the local representation of the stabilizer $K_{i}$ over the Pauli basis does not contain any $\sigma_{z}^{(j)}$ term in the $j$-th position but only identities $\mathbb{I}^{(j)}$ and the local representation of stabilizer $K_{j}$ does not contain any $\sigma_{z}^{(i)}$ term in the $i$-th position but only identities $\mathbb{I}^{(i)}$. One local measurement setting, composed of two measurements of kind $X$ to be performed on qubits $i$ and $j$ and measurements of type $Z$ to be performed on the remaining qubits, is then enough to measure the expectation values of the two stabilizers. When compositions of two or more stabilizers are concerned, Pauli matrices of kind $\sigma_{y}$ appear and local meas- ures of kind $Y$ are required. The connectedness hypothesis grants, for each stabilizer $K_{i}$, the existence of at least one stabilizer $K_{j}$, such that their composition $K_{i} K_{j}$ generates Pauli matrices of kind $\sigma_{y}$ in positions $i$ and $j$. We conclude that, on the one hand, witness $\tilde{W}_{n}$ needs a number of local measurement settings lower or equal to $n$. On the other hand, the number of local measurement settings required by witness $W_{n}$ is lower or equal to $\sum_{k=1}^{n} 2^{k-1}\left(\begin{array}{l}n \\ k\end{array}\right)=\frac{3^{n}-1}{2}$ but strictly greater than the number of local measurement settings required by the witness $\tilde{W}_{n}$.
[1] R. Jozsa and N. Linden, Proc. R. Soc. Lond. A 459, 2011 (2003).

[2] M. Van den Nest, preprint quant-ph/1204.3107 (2012).

[3] D. Bruß and C. Macchiavello, Phys. Rev. A 83, 052313 (2011).

[4] R. Qu, J. Wang, Z. Li and Y. Bao, Phys. Rev. A 87, 022311 (2013).

[5] M. Rossi, M. Huber, D. Bruß and C. Macchiavello, New J. Phys. 15, 113022 (2013).

[6] M. Hein, J. Eisert and H.-J. Briegel, Phys. Rev. A 69, 062311 (2004).

[7] C. Kruszynska and B. Kraus, Phys. Rev. A 79, 052304 (2009).

[8] M. Rossi, D. Bruß and C. Macchiavello, Phys. Scr. T160, 014036 (2014).

[9] M. Gachechiladze, C. Budroni and O. Gühne, Phys. Rev. Lett. 106, 070401 (2016).

[10] M. Hillery, V. Buzek and A. Berthiaume, Phys. Rev. A 59, 1829 (1999).

[11] M. Epping, H. Kampermann, C. Macchiavello and D. Bruß, arXiv:1612.05585

[12] D. Bruß, G. M. D'Ariano, M. Lewenstein, C. Macchiavello, A. Sen De, and U. Sen, Phys. Rev. Lett. 93, 210501 (2004).

[13] M. Hein, W. Dür, J. Eisert, R. Raussendorf, M. Nest and H.J. Briegel, arXiv preprint quant-ph/0602096.

[14] O. Gühne, M. Cuquet, F.E.S. Steinhoff, T. Moroder, M. Rossi, D. Bruß, B. Kraus and C. Macchiavello, J. Phys. A 47, 335303 (2014).

[15] O. Gühne and G. Tóth, Phys. Rep. 474, 1 (2009).

[16] R. Horodecki, P. Horodecki, M. Horodecki and K. Horodecki, Rev. Mod. Phys. 81, 865 (2009).

[17] M. Bourennane, M. Eibl, C. Kurtsiefer, S. Gaertner, H. Weinfurter, O. Gühne, P. Hyllus, D. Bruß, M. Lewenstein and A. Sanpera, Phys. Rev. Lett. 92, 087902 (2004).

[18] M. Horodecki, P. Horodecki, Paweł and R. Horodecki, Phys. Lett. A 223, 1 (1996).

[19] R.A. Horn and C.H. Johnson, Matrix Analysis, Cambridge University Press (2012).

[20] L.K. Grover, in Proceeding of the 28th Annual Symposium on the Theory of Computing (ACM Press, New York, 1996), pp. 212-219; L.K. Grover, Phys. Rev. Lett. 79, 325 (1997).

[21] M. Rossi, D. Bruß and C. Macchiavello, Phys. Rev. A 87, 022331 (2013).

[22] M.A. Nielsen, Phys. Rev. Lett. 83, 436 (1999).
[23] O. Gühne, P. Hyllus, D. Bruß, A. Ekert, M. Lewenstein, C. Macchiavello and A. Sanpera, Phys. Rev. A 66, 062305 (2002).

[24] G. Tóth and O. Gühne, Phys. Rev. Lett. 94, 060501 (2005).

[25] G. Tóth and O. Gühne, Phys. Rev. A 72, 022340 (2005).

[26] The sum of the binomial coefficient $\left(\begin{array}{c}k \\ k \prime\end{array}\right)$ over $k^{\prime}$ even is equal to the sum over $k^{\prime}$ odd $\left(0 \leq k^{\prime} \leq k\right)$, which is equal to half of the total sum. This equality is a direct consequence of the following identity:

$$
\sum_{k^{\prime}=0}^{k}(-1)^{k^{\prime}}\left(\begin{array}{c}
k \\
k^{\prime}
\end{array}\right) \equiv 0 .
$$

